\title{
Alteration in Hepatitis B Serology in Children Receiving Chemotherapy
}

\author{
Sebahat $\mathrm{Cam}^{1}$ and Mustafa Asim Yoruk ${ }^{2}$
}

\begin{abstract}
Chemotherapy-induced immunosuppression can lead to hepatitis B virus (HBV) reactivation in cancer patients. Both HBV carriers and individuals with serological signs of previously resolved HBV exposure are under the risk of severe hepatitis and liver failure during and after chemotherapy. The objective of this largest retrospective study was to analyze the consequences of HBV status in children receiving chemotherapy. A total of 479 patients ( 273 boys and 206 girls) aged 1-211 months diagnosed with acute hematologic malignancies and solid tumors were included in the study. Serological markers for HBV before and after chemotherapy and clinical data of the patients were evaluated retrospectively. Two hundred thirty-four of the participants were found to have protective antibody titers to HBV at admission. Five children were carrying HBV before chemotherapy. They received antiviral therapy during treatment and no reactivation was detected. Antibody against hepatitis B surface antigen (antiHBs) remained positive in 194 patients after chemotherapy. However, $17.09 \%$ (40/234) lost antiHBs positivity. In this group, three patients (1.28\%) who initially had positive antiHBs and antihepatitis B core antibody experienced HBV reactivation and lost their protective antiHBs at the end of the therapy. Median antiHBs titer significantly decreased after chemotherapy (213.14 [range: 24-888] vs. 180.85 [range: $0-850])(p=0.0094)$. The current relatively large trial demonstrated that protective antibody titers remarkably altered after chemotherapy, and at least $17 \%$ of the pediatric oncology cases lost antiHBs positivity. Therefore, vaccine prevention and close monitoring of serology should be considered during chemotherapy.
\end{abstract}

Keywords: children, cancer, hepatitis B, chemotherapy

\section{Introduction}

$\mathbf{H}$ EPATITIS B VIRUS (HBV) infection remains a common health problem all over the world despite the widespread use of vaccination. Nevertheless, a significant number of children are still infected each year (16). In our country, hepatitis B vaccination was included in the national vaccination program since 1998 and a decline in prevalence has been observed. The overall prevalence among 0-15-year-old children was dropped from $5.9 \%$ to $2.8 \%$ (8). However, a rate of about $3 \%$ still yields a significant number of HBV infections in children.

Immunosuppression caused by chemotherapy can lead to HBV reactivation. This can lead to interruption to chemotherapy as well as problems caused by the infection $(3,12)$. It is presently well known that anticancer treatment can interfere with the host immune system with the potential to cause viral reactivation in both hepatitis B surface antigen (HBsAg) positive patients and individuals with serological signs of previously resolved HBV exposure $(3,12,18)$. Recent literature data showed that prophylactic antiviral therapy is beneficial in cancer patients at risk of HBV reactivation (11). Therefore, HBV screening is recommended in many guidelines before immunosuppressive events are initiated $(5,10)$. However, the data on the course of hepatitis B infection during and after chemotherapy in children with cancer and changes in serology are limited.

In this study, we aimed to assess the pre- and posttreatment HBV serology, as well as the antibody titers in children receiving chemotherapy as the largest trial in literature. We also evaluated the course and outcome of the patients who were HBV carriers on admission and patients with HBV reactivation after chemotherapy.

\footnotetext{
${ }^{1}$ Division of Pediatric Gastroenterology, Department of Pediatrics, School of Medicine, Istanbul Medeniyet University, Istanbul, Turkey.
}

${ }^{2}$ Division of Pediatric Hematology and Oncology, Department of Pediatrics, Yeditepe University Hospital, Istanbul, Turkey. 


\section{Materials and Methods}

\section{Study group}

This study was carried out retrospectively at a tertiary reference university hospital. The medical records of all subsequent patients treated at the Department of Pediatric Hematology and Oncology between January 1996 and August 2018 were retrospectively examined in terms of HBV serology. The study was conducted in accordance with the Helsinki Declaration as revised in 2013. Children diagnosed with acute leukemia, lymphoma, and solid tumors were included in the study. HBsAg, antibodies against $\mathrm{HBsAg}$ (antiHBs), and antibodies against hepatitis B core antigen (antiHBc) were examined using enzyme-linked immunosorbent assay methods. Antibody titers $>10 \mathrm{mIU} / \mathrm{mL}$ were considered antiHBs positive. For immunocompromised people including receiving cytotoxic therapies anti-HBs concentrations $<10 \mathrm{mIU} / \mathrm{mL}$ was the critical titer level (7). Positivity in hepatitis B serology markers and antibody levels against hepatitis B were routinely assessed both at the time of diagnosis and 6 months after completion of chemotherapy. $\mathrm{HBV}$ reactivation was accepted as a sudden and rapid increase in HBV DNA level or the de novo detection of HBV DNA viremia whenever undetectable before the initiation of the immune-suppressive, cytotoxic, or biological modifier therapy (7).

Serological markers were compared before and after treatment; effects of age, gender, and underlying disease on antibody titers level were evaluated. Also, we performed a subgroup analysis regarding the vaccination status of the children. A national vaccination program was started in 1998. Hepatitis B vaccine is administered three times with the schedule of " 0,1 , and 6 months." The first vaccine is administered on the day of birth or in the next few days after birth.

The treatment protocols of the patients were as follows: Medulloblastoma patients received the Children's Oncology Group (COG) study ACNS-0331 protocol, acute lymphoblastic leukemia (ALL) patients had ALL-BerlinFrankfurt-Münster (BFM)-2000 (modified BFM-95) and ALL-Intercontinental Berlin-Frankfurt-Münster (IC-BFM)2009 protocols. Acute myeloid leukemia (AML) patients received AML-BFM-93, AML-BFM-98, and AML-BFM-04 protocols. Hodgkin lymphoma patients had doxorubicin/bleomycin/vinblastine/dacarbazine (ABVD) regimen. Patients with mature B cell lymphoma/leukemia had nonHodgkin Lymphoma (NHL)-BFM-95 protocol. Lymphoblastic lymphoma patients had NHL-BFM 95, and specific T cell lymphomas (EURO-LB-02) protocols. Neuroblastoma cases had Turkish Pediatric Oncology Group (TPOG)Neuroblastoma (NBL)-2003, and TPOG-NBL-2009 protocols. Patients with rhabdomyosarcoma and soft tissue sarcomas received Cooperative Weichteilsarkom Studiengruppe (CWS)-guidance protocols. Wilms tumor patients had National Wilms Tumor Study (NWTS)-5 protocol, patients with germ cell tumor had bleomycin/etoposide/ cisplatin (PEB) regimen. The types of chemotherapeutic agents used in these protocols were alkylating agents (cyclophosphamide, ifosfamide, dacarbazine, carboplatin, cisplatin, and lomustine), antimetabolites (cytosine arabinoside, 6mercaptopurine, methotrexate, and 6-thioguanine), antitumor antibiotics (mitoxantrone, bleomycin, and dactinomycine), topoisomerase II inhibitors (daunorubicin, doxorubicin, etoposide, and idarubicin), mitotic inhibitors (vincristine, vinblastine, and vindesine), enzymes (1-asparaginase), corticosteroids, and alltrans retinoic acid.

Antiviral prophylaxis (Lamivudine) was started 1 week before chemotherapy to children with HBsAg positivity at the time of diagnosis, continued throughout the treatment, and for at least 6 months after the end of the last cycle. Liver function tests and HBV-DNA were followed every 3 months in all patients.

\section{Statistical analysis}

Statistical Package for Social Sciences (SPSS) version 20.0 for Windows (SPSS, Inc., Chicago, IL, USA) was utilized for statistical analysis. The Mann-Whitney $U$ test and the chi-square test were used to compare between variables and a $p$-value $<0.05$ was considered statistically significant. Data are presented as numbers and percentages or medians and ranges, as appropriate. The chi-square test was used for evaluating categorical values.

\section{Results}

A total of 479 patients (273 boys and 206 girls) were included in the study. The mean age of the patients was $81.49 \pm 62.04$ months. The characteristics of the patients are shown in Table 1 . One hundred thirty patients had been treated for leukemia, 108 for lymphomas, and 241 for solid tumors.

Previous vaccination of the patients had been carried out according to the national vaccination program, which was started in 1998. One hundred forty-five of our patients were born before 1998. None of the children born before 1998 were vaccinated for hepatitis B. In this group antiHBs antibody positivity was present in only nine patients $(6.2 \%)$. In contrast, $67.3 \%$ of the patients who were born after 1998 were antiHBs positive. Five patients were $\mathrm{HBsAg}$ positive at the time of diagnosis. These HBsAg positive five patients all were born before 1998 and not vaccinated for HBV. Antiviral prophylaxis (Lamivudine) was given to these patients during chemotherapy. Hepatitis $\mathrm{B}$ reactivation was not detected in any patient during or after chemotherapy. Protective antibody titers against hepatitis B were detected in 234 patients $(48.8 \%)$ at admission. Two hundred forty-five $(51.2 \%)$ were negative for antiHBs antibody.

After chemotherapy, 277 patients were HBsAg and antiHBs negative, 194 patients were $\mathrm{HBsAg}$ negative, antiHBs positive. Consequently, it was shown that $17.09 \%(40 / 234)$ of the cases lost antiHBs positivity. Only, 3 out of 234 patients $(1.28 \%)$ with initial positive antiHBs and antiHBc experienced hepatitis $\mathrm{B}$ reactivation and lost their protective antiHBs at the end of the therapy. Hepatitis $B$ reactivation was confirmed by detection of HBV DNA in all these cases. Two of these patients were acute leukemia, one patient diagnosed as Wilms tumor. The alteration in HBV serology is summarized in Figure 1.

The course of the patients with regard to the vaccination is presented in Figure 2. The figure detailed the subgroups "born before 1998" and "born after 1998." A total of 250 children had hepatitis B vaccination after 1998.

A total of 130 children had anti-HBc measurement before the chemotherapy. Only 14 children had positive anti-HBc results. All these 14 children born before 1998 and did not receive $\mathrm{HBV}$ vaccine. It was also documented that five of 
Table 1. Characteristics and Demographic Data of the Patients According to Serology Results at The Time of Diagnosis

\begin{tabular}{lccc}
\hline Variable & $\begin{array}{c}\text { All children } \\
(\mathrm{n}=479)(100 \%)\end{array}$ & $\begin{array}{c}\text { AntiHBs Ab }>10 \mathrm{mIU} / \mathrm{mL} \\
(\mathrm{n}=234)(48.8 \%)\end{array}$ & $\begin{array}{c}\text { AntiHBs } \mathrm{Ab}<10 \mathrm{mIU} / \mathrm{mL} \\
(\mathrm{n}=245)(51.2 \%)\end{array}$ \\
\hline Gender & & & 137 \\
$\quad$ Male & 273 & 136 & 108 \\
$\quad$ Female & 206 & 98 & 83 \\
Age at diagnosis & 162 & 79 & 77 \\
$\quad<72$ Months & 150 & 73 & 85 \\
73-144 Months & 167 & 82 & 6.626 \\
144-216 Months & & & 59 \\
Malignancy types & 130 & 65 & 59 \\
$\quad$ Acute leukemia & 108 & 49 & 121 \\
$\quad$ Lymphoma & 241 & 120 & 0.712 \\
Solid tumors & &
\end{tabular}

antiHBs, antibody against hepatitis B surface antigen.

these cases were HBsAg positive and received antiviral therapy. In the vaccinated group 37 children lost antiHBs positivity after chemotherapy.

Hepatitis B antibody titers were remarkably altered after chemotherapy. Median antiHBs titer after chemotherapy were significantly lower than those before therapy $(213.14$ [range: $24-888$ ] vs. 180.85 [range: $0-850$ ] $)(p=0.0094)$. In
40 children with protective antibody levels before therapy, antiHBs titers decreased below the protection level after chemotherapy. When the change in antiHBs titer was examined by gender, age, and disease type, no significant difference was detected between the groups (Table 2). Except for three patients with reactivation, liver enzymes were normal in all patients.

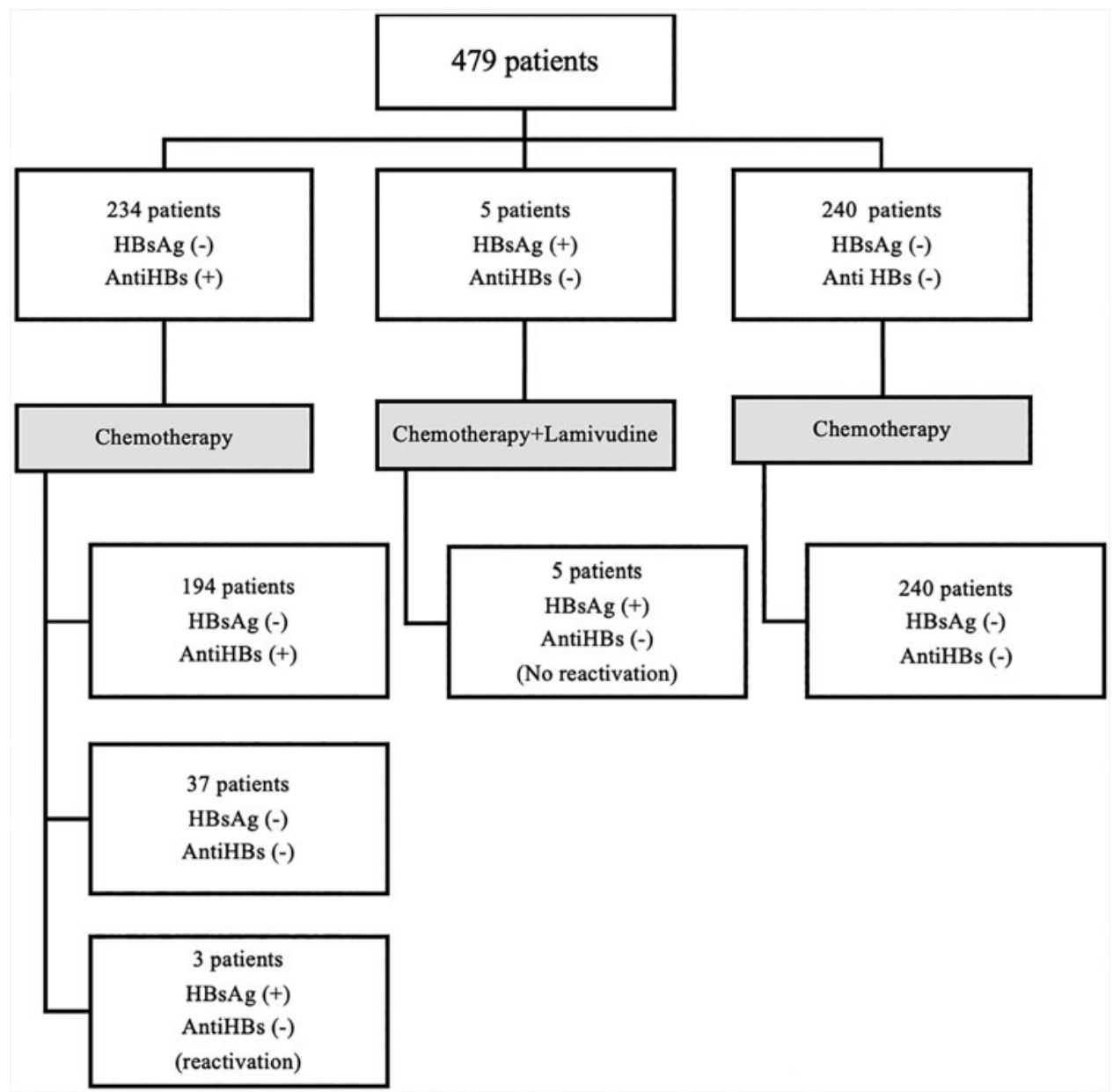

FIG. 1. HBV serologies of patients before and after treatment. HBV, hepatitis B virus. 


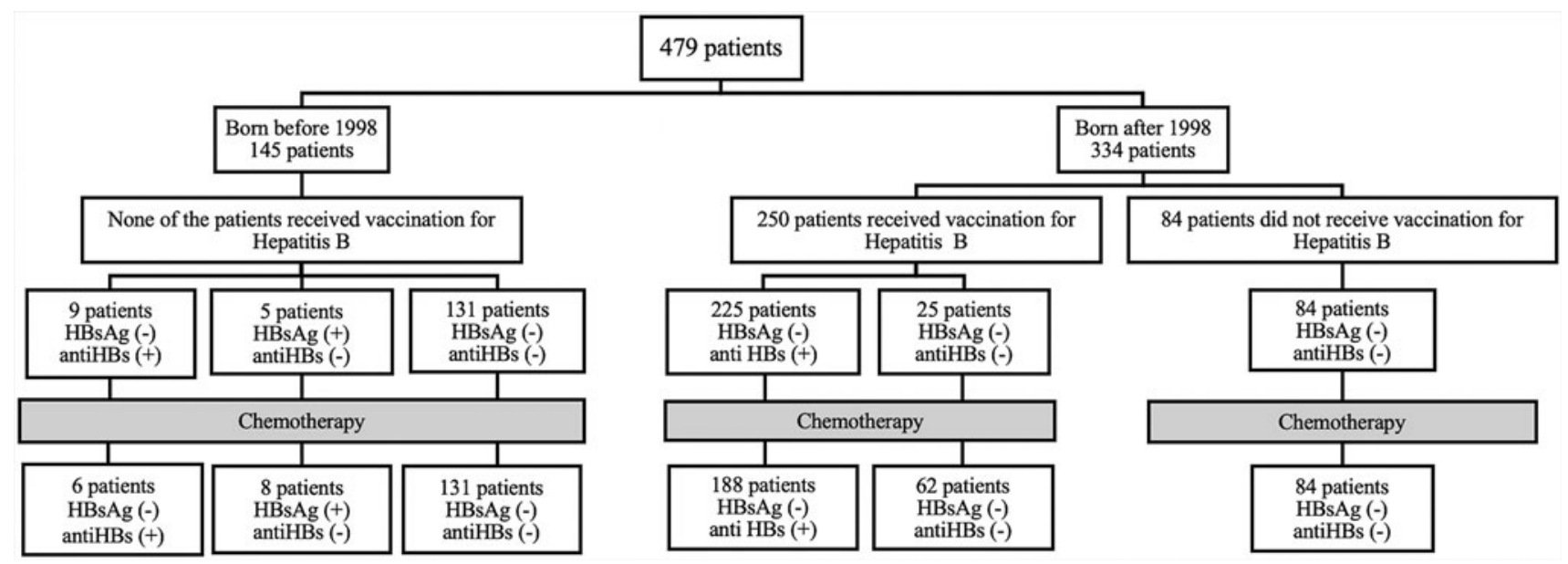

FIG. 2. The distribution of the patients with respect to vaccination status.

\section{Discussion}

Although vaccination causes a significant decrease in the prevalence of HBV infection, the virus can still lead to serious complications, particularly in immunocompromised patients. Therefore, in patients undergoing chemotherapy, pretreatment serological screening and prophylaxis are important and highly recommended in various guidelines (10,11). Systemic chemotherapy can cause a marked deterioration in immune function. The underlying mechanism in the reduction of antibody titers after chemotherapy is probably the destruction of antibody-producing B lymphocytes by chemotherapy. Caver et al. observed B cell lymphopenia in 16 patients who were receiving maintenance treatment for ALL (2). Significant decreases in intramedullary plasma cell count also have been observed during chemotherapy for ALL (14). Mustafa et al. showed that children with malignancy had specific and nonspecific immune changes after chemotherapy and antibody responses to immunization were still defective 12 months after

Table 2. Distribution of Patients Regarding the Loss of Hepatitis B Protective Antibody By Age, Gender, And Disease Type

\begin{tabular}{lccc}
\hline & $\begin{array}{c}\text { Loss of } \\
\text { antiHBs } \\
\text { antibody } \\
(\mathrm{n}=40)\end{array}$ & $\begin{array}{c}\text { No loss of } \\
\text { antiHBs } \\
\text { antibody } \\
(\mathrm{n}=194)\end{array}$ & $\mathrm{p}$ \\
\hline Gender & 25 & 111 & 0.537 \\
$\quad$ Male & 15 & 83 & \\
$\quad$ Female & & & \\
Age at diagnosis & 8 & 71 & 0.338 \\
$\quad<72$ Months & 8 & 67 & \\
73-144 Months & 24 & 56 & \\
144-216 Months & & & \\
Malignancy types & 11 & 54 & 0.982 \\
$\quad$ Acute leukemia & & & \\
$\quad(n=65)$ & 8 & 41 & \\
$\quad$ Lymphoma $(n=49)$ & 21 & 99 & \\
$\quad$ Solid tumors $(n=120)$ & & & \\
\hline
\end{tabular}

the end of treatment especially in younger patients (13). Therefore, a serological screening program also makes sense during chemotherapy. HBV reactivation has previously been reported in $\mathrm{HBsAg}$ positive cancer patients receiving systemic chemotherapy $(3,19)$. Patients with resolved infection are also at risk of reactivation (18). In patients with resolved infection, a decrease in HBsAb titer usually occurs during or after discontinuation of chemotherapy, and the subsequent emergence of HBsAg and HBV DNA leads to $\mathrm{HBV}$ reactivation (6).

There are few studies about post-treatment changes in HBV serology in pediatric oncology patients and the course of HBsAg positive patients during systemic chemotherapy. The effects of chemotherapy on the course of antiHBs titer in children receiving chemotherapy due to malignancies have not been investigated in detail. We retrospectively analyzed the changes in hepatitis B serology and antiHBs titers in our patients before and after receiving chemotherapy for pediatric malignancies. When hepatitis B carriers were excluded, antiHBs positivity was present in almost half of the patients at admission. One hundred forty-five of our patients were born before 1998 when the hepatitis B vaccine was not in the national vaccination program. In this group, antiHBs antibody positivity was present in only nine patients $(6.2 \%)$. Approximately $70 \%$ of the patients born after 1998 were antiHBs positive on admission. These data show the effectiveness of the vaccination. In a previous study with pediatric sarcoma patients, Yu et al. showed that the majority of patients did not have detectable antibody titers against hepatitis B after chemotherapy (20). Of the 101 patients whose hepatitis B surface antibody titers were measured, 65 (64.4\%) had negative antibody titers. In this study, pretreatment values were not specified. In our study, the rate of children with a lack of protective antibody for hepatitis B was slightly lower than reported by $\mathrm{Yu}$ et al. We observed that about $17 \%$ of the patients lost antiHBs positivity after chemotherapy. In a smaller group, Baytan et al. demonstrated that 25 out of 40 children $(62.5 \%)$ with positive antiHBs titers lost their immunity at mean $15.9 \pm 10$ months (range 6-21 months) during chemotherapy (1).

At the same time, in this study, it was found that protective antibody levels for hepatitis B further decreased 
significantly after treatment compared with levels before treatment. A decrease in antiHBs titer after exposure to chemotherapy was observed in children with malignancy regardless of age, gender, and disease type. Our outcomes were similar to the results of Zignol et al., which state that postchemotherapy patients are likely to lose positive antibody titers to all vaccine-preventable diseases, including hepatitis B (22). They recorded the loss of protective antibody serum titers in $52 \%$ of pediatric cancer patients evaluated before and after chemotherapy. Keskin et al. in a group of 162 children with pediatric malignancies demonstrated that pre-existing immunity to measles, mumps, rubella, and hepatitis B may be lost after completion of chemotherapy (9).

Three patients who were HBsAg negative and antiHBs positive at the time of diagnosis were found to be $\mathrm{HBsAg}$ positive after chemotherapy. None of these children were vaccinated for HBV. All patients had positive $\mathrm{HBcIgG}$ values. These patients were thought to gain immunity through a "silent" HBV infection. Antiviral prophylaxis was not given to these patients. In contrast, at the time of diagnosis, five HBsAg positive patients were started on lamivudine and continued during chemotherapy. No liver dysfunction was observed in these patients. Prophylaxis was started 1 week before chemotherapy and continued for at least 6 months after the end of the last cycle as a similar way in the literature $(5,15)$. Data from other open studies suggest that lamivudine may be effective both in the treatment of $\mathrm{HBV}$ reactivation resulting from chemo/immunosuppressive therapy and in the prevention of reactivation in HBV carriers $(4,17,21)$. Despite the small number of patients receiving antiviral therapy results from our study suggest that lamivudine can be safely and effectively used in children with malignant diseases carrying HBV.

In patients born after 1998, when the national vaccination program began for HBV, immunity for HBV was higher as expected compared with those previously born. There was no HBV carrier at the time of diagnosis in patients born after 1998. Also, children with seroreversion from HBsAg negative to HBsAg positive state were born before 1998. This condition, which shows the effectiveness of HBV vaccination, is particularly important for oncology patients.

The limitations of this study are its retrospective nature, leading to the lack of information about HBV DNA status of the patients other than carriers at the beginning and patients with reactivation. Anti-HBc measurement was available in about one-third of the patients. The results indicated that a total of 14 patients underwent hepatitis B infection before the routine vaccination strategy in 1998. Since this was a retrospective study, anti-HBc results were unfortunately not available for all cases. Nevertheless, the consequences of the study suggest a requirement for follow-up of $\mathrm{HBs}$ status after chemotherapy. Also, to our knowledge, this is the study involving the largest group of children with a pediatric malignancy investigating the effect of chemotherapy on the course of hepatitis B serology and antiHBs titers in children. The follow-up of hepatitis B serology during and after chemotherapy is also a strong suggestion in a recent guideline (7).

In this study, we showed that protective antibody levels for hepatitis B decreased significantly after chemotherapy compared with those before treatment. In addition, a certain rate of children lost pre-existing humoral immunity against HBV. These patients are at risk for severe hepatitis B infection due to immunosuppression. Therefore, it can be concluded that children with malignancy should be screened and closely monitored for HBV infection before and after chemotherapy.

\section{Author Disclosure Statement}

No competing financial interests exist.

\section{Funding Information}

No funding was received for this article.

\section{References}

1. Baytan B, Gunes AM, and Gunay U. Efficacy of primary hepatitis B immunization in children with acute lymphoblastic leukemia. Indian Pediatr 2008;45:265-270.

2. Caver TE, Slobod KS, Flynn PM, et al. Profound abnormality of the $\mathrm{B} / \mathrm{T}$ lymphocyte ratio during chemotherapy for pediatric acute lymphoblastic leukemia. Leukemia 1998;12:619-622.

3. Chen CY, Huang SY, Cheng A, et al. High risk of hepatitis $B$ reactivation among patients with acute myeloid leukemia. PLoS One 2015;10:e0126037.

4. el-Sayed MH, Shanab G, Karim AM, et al. Lamivudine facilitates optimal chemotherapy in hepatitis B virusinfected children with hematological malignancies: a preliminary report. Pediatr Hematol Oncol 2004;21:145-156.

5. European Association for the Study of the Liver. EASL 2017 Clinical Practice Guidelines on the management of hepatitis B virus infection. J Hepatol 2017;67:370398.

6. Firpi RJ, and Nelson DR. Management of viral hepatitis in hematologic malignancies. Blood Rev 2008;22:117-126.

7. Indolfi G, Abdel-Hady M, Bansal S, et al. Management of Hepatitis B virus infection and prevention of Hepatitis virus reactivation in children with acquired immune deficiencies or undergoing immune suppressive, cytotoxic, or biological modifier therapies. J Pediatr Gastroenterol Nutr 2020;70: 527-538.

8. Kanra G, Tezcan S, Badur S; Turkish National Study Team. Hepatitis B measles seroprevalence among Turkish children. Turk J Pediatr 2005;47:105-110.

9. Keskin Yildirim Z, and Buyukavci M. Assessment of humoral immunity to hepatitis $\mathrm{B}$, measles, rubella, and mumps in children after chemotherapy. J Pediatr Hematol Oncol 2018;40:e99-e102.

10. Law MF, Ho R, CheungCK, et al. Prevention and management of hepatitis B virus reactivation in patients with hematological malignancies treated with anticancer therapy. World J Gastroenterol 2016;22:6484-6500.

11. Li H, Zhang HM, Chen LF, et al. Prophylactic lamivudine to improve the outcome of HBsAg-positive lymphoma patients during chemotherapy: a systematic review and meta analysis. Clin Res Hepatol Gastroenterol 2015;39: 80-92.

12. Loomba R, and Liang TJ. Hepatitis B reactivation associated with immune suppressive and biological modifier therapies: current concepts, management strategies, and future directions. Gastroenterology 2017;152:1297-1309.

13. Mustafa MM, Buchanan GR, Winick NJ, et al. Immune recovery in children with malignancy after cessation of chemotherapy. J Pediatr Hematol Oncol 1998;20:451-457. 
14. Nilsson A, De Milito A, Engström P, et al. Current chemotherapy protocols for childhood acute lymphoblastic leukemia induce loss of humoral immunity to viral vaccination antigens. Pediatrics 2002;109:e91.

15. Rossi G, Pelizzari A, Motta M, and Puoti M. Primary prophylaxis with lamivudine of hepatitis $\mathrm{B}$ virus reactivation in chronic HBsAg carriers with lymphoid malignancies treated with chemotherapy. $\mathrm{Br} \mathrm{J}$ Haematol 2001;115:58-62.

16. WHO. Global Hepatitis Report 2017. Geneva: World Health Organization, 2017. https://www.who.int/hepatitis/ publications/global-hepatitis-report2017/en/ (accessed October 16, 2020).

17. Xu Z, Dai W, Wu YT, et al. Prophylactic effect of lamivudine on chemotherapy-induced hepatitis $\mathrm{B}$ virus reactivation in patients with solid tumor: a meta-analysis. Eur J Cancer Care (Engl) 2018;27:e12799.

18. Yeo W, Chan TC, Leung NW, et al. Hepatitis B virus reactivation in lymphoma patients with prior resolved hepatitis B undergoing anticancer therapy with or without rituximab. J Clin Oncol 2009;27:605-611.

19. Yeo W, Zee B, Zhong S, et al. Comprehensive analysis of risk factors associating with Hepatitis B virus (HBV) reactivation in cancer patients undergoing cytotoxic chemotherapy. Br J Cancer 2004;90:1306-1311.
20. Yu J, Chou AJ, Lennox A, et al. Loss of antibody titers and effectiveness of revaccination in post-chemotherapy pediatric sarcoma patients. Pediatr Blood Cancer 2007;49:656660.

21. Zhang MY, Zhu GQ, Zheng JN, et al. Nucleos(t)ide analogues for preventing HBV reactivation in immunosuppressed patients with hematological malignancies: a network meta-analysis. Expert Rev Anti Infect Ther 2017; 15:503-513.

22. Zignol M, Peracchi M, Tridello G, et al. Assessment of humoral immunity to poliomyelitis, tetanus, hepatitis B, measles, rubella, and mumps in children after chemotherapy. Cancer 2004;101:635-641.

Address correspondence to:

Dr. Sebahat Cam

Division of Pediatric Gastroenterology

Department of Pediatrics

School of Medicine

Istanbul Medeniyet University

Kadikoy

Istanbul 34722

Turkey

E-mail: imamoglus@yahoo.com 